\title{
Être créatif avec les technologies : enseigner le français de spécialité au niveau Master dans les cours de langues étrangères à l'Université de Turin (UniTO)
}

Adriana Teresa Damascelli ${ }^{1}$ et Marie-Berthe Vittoz $^{2}$, Centre des Langues - Université de Turin, Italie

\section{Introduction}

Depuis le début des années quatre-vingt-dix, les nouvelles technologies multimédias, et en particulier les ressources numériques, caractérisent toutes les activités quotidiennes, aussi bien professionnelles que privées. Ce n'est que plus récemment que ces technologies se sont diffusées plus largement dans les pratiques pédagogiques (Hirschsprung et HoulonTrémolières $)^{3}$. On constate que l'intégration des technologies dans les activités de classe a modifié l'environnement pédagogique. Dans la didactique des langues étrangères, au niveau avancé par exemple, l'existence et la diffusion des ressources telles que les logiciels pour l'analyse des données linguistiques ou la traduction assistée par ordinateur (TAO) permettent le déroulement d'activités d'enseignement avec un usage stratégique de plusieurs outils et applications numériques, ayant des fonctions à la fois cognitives, collaboratives et créatives.

Cet article explore l'hypothèse que l'introduction de modules linguistiquesinformatiques dans les cours de langues des parcours de FLE au niveau Master peut promouvoir l'apprentissage d'une façon plus consciente finalisé à l'analyse de la langue et aussi à l'élaboration de stratégies pour la gestion de la langue dans des contextes de communication et aussi de médiation.

\section{Cadre théorique}

La créativité est un sujet auquel plusieurs disciplines du savoir ont consacré beaucoup d'études et ont proposé diverses théories et définitions. La vision dominante affirme que la créativité est connotée par les quatre $« \mathrm{P} »$, process (processus), product (produit), person (individu) et place (Kozbelt et al.). ${ }^{4}$ Dans son livre Imagination and Creativity in Childhood, Lev Vygotsky développe sa théorie de la créativité comme un processus conscient où l'émotion, la pensée et l'imagination entrent en relation. Selon Vygotsky, l'être humain, y compris les enfants, sont créatifs et la créativité constitue la base pour l'art, la science et la technologie.

$\mathrm{Au}$ cours du temps, diverses approches de la créativité en ont fourni plusieurs définitions, chacune étant élaborée selon les facteurs pris en considération. Plus récemment, 
d'après le psychologue Mihaly Csikszentmihalyi, « la créativité est la capacité à exprimer des pensées inhabituelles qui sont intéressantes et enrichissantes, à percevoir le monde d'une façon critique, par des jugements pénétrants qui mènent à des découvertes significatives » (25). D'autre part, la définition proposée par Nathalie Bonnardel, à savoir « la capacité à produire une idée exprimable sous une forme observable ou à réaliser une production, qui soit à la fois novatrice et inattendue, adaptée à la situation et considérée comme ayant une certaine utilité ou de la valeur » (95), en souligne le caractère de nouveauté et d'originalité. De plus, les travaux présentés soulignent l'existence d'une relation entre les aspects individuels et les aspects environnementaux. Mais, pourquoi la créativité reçoit-elle autant d'attention de la part des chercheurs ? L'idée sous-jacente est que le développement d'une pensée créative peut aider à l'acquisition de capacités utiles à la résolution des problèmes (problem-soving abilities). Selon le Conseil de l'Europe, l'introduction de méthodologies et/ou situations d'apprentissage qui favorisent le développement de compétences et de la créativité sont nécessaires à tous les niveaux de l'éducation et de la formation en général. Le Conseil de l'Europe reconnaît que

... schools have a duty to provide their pupils with an education which will enable them to adapt to an increasingly globalised, competitive, diversified and complex environment, in which creativity, the ability to innovate, a sense of initiative, entrepreneurship and a commitment to continue learning are just as important as the specific knowledge of a given subject. (Cachia et al. 25)

Donc, à travers la créativité les apprenants peuvent mieux se confronter avec le monde du travail et avec la situation complexe de la société moderne qui, aujourd'hui, exigent des connaissances spécialisées et des capacités hétérogènes surtout orientées vers le numérique pour pouvoir répondre le mieux possible aux attentes professionnelles, qui désormais exigent une « litteratie numérique » (Lankshear et Knobel).

La valeur que la créativité possède, en tant que moteur de l'innovation dans tous les domaines de la vie humaine, a suggéré à l’Union européenne de déclarer 2009 «Année européenne de la créativité et de l'innovation » et de procéder à des initiatives visant à soutenir les programmes d'échange et la mobilité6.

Le document « Décision du Parlement Européen et du Conseil » du 16 décembre 2008 en spécifie les objectifs particuliers en soulignant les facteurs qui contribuent à favoriser la créativité et la capacité d'innovation, comme, par l'exemple, « créer un environnement propice à l'innovation, et à la capacité d'adaptation dans un monde en rapide évolution, [...] développer la communication interculturelle, [...] promouvoir l'enseignement des compétences basiques et approfondies des technologies, [...] sensibiliser le public, que les connaissances et la flexibilité sont importantes, $[\ldots] \gg .{ }^{7}$ 
Vu comme un processus qui génère des idées nouvelles, des expressions et des formes qui peuvent élargir et conduire à de nouvelles façons d'utiliser les connaissances, la créativité est considérée comme une compétence clé transversale pour l'apprentissage. Elle constitue un élément important pour parvenir aux objectifs prévus au terme d'un processus d'apprentissage «learning outcomes ». Ces objectifs sont contenus dans le document «Les Descripteurs de Dublin » et sont répartis en cinq catégories d'acquis selon chaque cycle éducatif. ${ }^{8}$ La créativité également favorise le développement des huit compétences clés introduites par le Parlement européen et le Conseil de l'Europe (Parlement européen et le Conseil)

Par conséquent, il est nécessaire que les futurs enseignants comprennent l'importance de la créativité et les effets positifs sur l'apprenant. À ce propos, comme le souligne National Curriculum Handbook du Royaume Uni, 2004 :

By providing rich and varied contexts for pupils to acquire, develop and apply a broad range of knowledge, understanding and skills, the curriculum should enable pupils to think creatively and critically, to solve problems and to make a difference for the better. It should give them the opportunity to become creative, innovative, enterprising and capable of leadership to equip them for their future lives as workers and citizens. It should enable pupils to respond positively to opportunities, challenges and responsibilities, to manage risk and cope with change and adversity.

Pour ce faire, on peut également utiliser les applications numériques disponibles pour tous et même pour les plus jeunes qui l'utilisent déjà dans leur quotidien. En fait, l'arrivée de la technologie dans la vie domestique a permis de partager des matériaux et des ressources et, en même temps, aux communautés de partager et de rendre accessibles leurs propres valeurs culturelles, compétences et aptitudes (Silverstone). Nombreuses sont les potentialités offertes à leurs utilisateurs, il suffit de penser aux applications d'informatique sociale / social computing applications:

Social computing applications vary from social networking sites (like Facebook; MySpace); sharing of bookmarks (del.icio.us; Citeulike); sharing of multimedia (Flickr; YouTube), online gaming (Second Life) and blogging, to mention but a few. These applications offer new opportunities for people to express their creativity, make it available to a large audience and get feedback and recognition. (Edwards 125)

Dans un article paru en 2012 dans le journal britannique The Guardian, l'auteur Chaz Pugliese montre comment la créativité ne doit pas être conçue comme une «option » pour le professeur de langue, mais bien comme le meilleur des outils à sa disposition pour motiver les apprenants à apprendre. Toutefois, la créativité doit être recherchée et nourrie à travers la réflexion sur les enjeux que la société contemporaine présente dans toutes les situations. 
L'éducation, par exemple, est une situation dans laquelle la réalisation de la transmission du savoir représente un défi. De même, dans l'éducation linguistique, les objectifs de compétence et conscience linguistique qui doivent être atteints pour qu'un parcours soit efficace, constituent une source de stimulation continue. Comment ces objectifs peuvent-ils être réalisés ? Quels outils peuvent aider et de quelle manière ? Un des enjeux «créatifs » dans l'éducation linguistique, en particulier L2, se situe au niveau digital.

\section{Méthodologie expérimentale}

Les technologies de l'information et de la communication dans l'enseignement (TICE) ont profondément modifié l'éducation. Elles permettent de penser à une didactique plus dynamique où les méthodes traditionnelles et innovatrices peuvent coexister et s'intégrer pour créer des environnements d'apprentissage efficaces. Ces contextes deviennent essentiels et représentent un défi pour l'éducation, habituellement conçue comme l'ensemble de notions acquises selon un processus linéaire et cumulatif (Rey et Feyfant). Pour autant, être créatif dans l'enseignement signifie adopter des stratégies innovatrices qui permettent, en même temps, de développer de nouvelles méthodes, de nouveaux outils et de nouveaux contenus au bénéfice des élèves (Ferrari et al.) et de générer auprès des étudiants la créativité, une compétence que la Commission européenne considère comme compétence clé pour une formation tout au long de la vie. En effet, grâce à ces stratégies, on peut impliquer les étudiants dans des activités qui leur permettent de développer la « capacité à proposer de nouvelles solutions, de nouvelles visions pertinentes des choses » (Taddei). Mais alors comment peut-on être créatif dans l'enseignement des langues étrangères ? Dans la littérature scientifique, on constate que les technologies sont souvent considérées comme des outils efficaces, à condition que les acteurs, enseignants et étudiants, soient numériquement compétents.

\section{La linguistique de corpus et la traduction assistée par ordinateur (TAO)}

Dans le domaine de la didactique des langues étrangères l'introduction de la linguistique de corpus dans les cours de langues constitue un exemple de créativité appliquée à l'utilisation d'outils professionnels pour l'analyse de la langue. En effet, la linguistique de corpus est née comme méthodologie pour analyser et tester des hypothèses dans la langue. ${ }^{9} \mathrm{~A}$ travers une interface d'interrogation les chercheurs peuvent définir une recherche et obtenir un certain nombre de résultats qui leur permet de résoudre un problème linguistique précis.

Grâce aux contributions de plusieurs chercheurs (Hunston), la linguistique de corpus a conquis sa place dans la didactique des langues. On s'est aperçu que, si les étudiants mêmes sont mis en condition d'être des chercheurs, ils peuvent acquérir des méthodes appropriées de 
réflexion pour réfléchir sur la langue et ses phénomènes. En fait, les outils proposés par les logiciels d'analyse du corpus permettent aux étudiants d'observer les phénomènes de la langue et de faire des généralisations, des hypothèses à différents niveaux pas seulement liés à l'usage contextuel, mais aussi d'explorer des connotations culturelles et pragmatiques.

De la même façon, la traduction assistée par ordinateur qui est conduite par des traducteurs professionnels peut être incluse dans la didactique des langues. En effet, à travers l'utilisation de «logiciels dédiés », les étudiants peuvent réfléchir sur les problèmes de médiation d'une langue à l'autre et considérer les aspects liés à la localisation ${ }^{10}$. Ces logiciels sont caractérisés, en principe, par :

a. un environnement de traduction où les traducteurs créent ou utilisent les mémoires de traduction qui sont des bases de données contenant des segments de texte dans la langue source et celle d'arrivée ;

b. un environnement pour la création et utilisation de bases de données terminologiques ;

c. un environnement pour l'alignement où l'on compare la traduction d'un texte avec la version dans la langue source pour associer les segments correspondants, les lier comme unités de traduction et les insérer dans la mémoire de traduction ${ }^{11}$.

Description du contexte et des activités de l'expérimentation

Pour la réalisation de l'expérimentation on a pris en considération les étudiants qui fréquentent le cours de langue française du Master en Langues pour la Communication Internationale au Département de «Lingue e Letterature Straniere e Culture Moderne ». Le choix était motivé par deux raisons : la première liée aux compétences générales que ces étudiants ont acquis grâce à la formation linguistique reçue pendant les cours des trois années de licence ${ }^{12}$; la deuxième raison est liée au contexte d'acquisition du cours master choisi où les étudiants pourront acquérir des compétences diversifiées sur la langue aussi bien linguistiques que culturelles, nécessaire pour travailler dans un contexte international.

Les activités se sont déroulées tout au long d'un module de vingt heures intitulé « L'étude de la langue des institutions internationales dans un environnement numérique technologique » et conduites dans un des laboratoires informatiques du Centre des Langues de l'Université de Turin doté de vingt-cinq ordinateurs avec l'accès à Internet, de logiciels pour le traitement automatique des corpus, tel que SketchEngine, et pour la traduction assistée par ordinateur, en particulier Trados.

Les objectifs de ce module étaient multiples : d'abord, comprendre comment une approche créative peut réélaborer l'utilisation des technologies disponibles d'une façon efficace pour aider les étudiants à une réflexion sur la langue ; ensuite, proposer des activités 
pratiques pour l'analyse de la langue des sites institutionnels pour produire du matériel sur lequel réfléchir ; enfin, évaluer les connaissances et compétences numériques des étudiants. Et encore, apprendre à utiliser les technologies et Internet comme ressources pour résoudre les problèmes de traduction et d'usage.

Avant de commencer les activités prévues dans le module, on a planifié deux sessions introductives de deux heures chacune, la première sur la traduction assistée par ordinateur et la deuxième pour la description des tâches pratiques. Pendant la première rencontre, les étudiants se sont familiarisés avec les concepts clés de la traduction assistée par ordinateurs, comme mémoire de traduction, localisation, et banque terminologique. Lors de la deuxième rencontre, les objectifs du module et les moyens pour réaliser les activités de recherche et d'analyse de la langue ont été présentés aux étudiants. Par la suite, les sites institutionnels UNESCO, FAO, Conseil de l'Europe, ONU ont été proposés comme sources pour la collecte d'informations linguistiques sous la forme de corpus. Ces sites offrent des textes économiques-juridiques dans plusieurs domaines et, donc, des exemples de langue spécifique liée à la communication dans des contextes internationaux.

Pour la réalisation des objectifs de ce module, on a demandé aux étudiants de collecter des corpus de vingt mille mots sur un des sujets identifiés dans un des sites proposés. En même temps on a préparé des corpus de référence pour le français, l'italien et l'anglais, étant donné que le cours était ouvert aussi aux étudiants Erasmus. ${ }^{13}$ Les étudiants ont travaillé pour la collecte des textes nécessaires à l'analyse. ${ }^{14}$

Au terme de cette première phase on a consacré deux heures à introduire la linguistique de corpus, qui est une méthodologie née pour mener des recherches sur les phénomènes linguistiques aux différents niveaux de la langue, mais maintenant très diffuse dans l'enseignement des langues étrangères. Cette méthodologie, en effet, d'une façon transversale, est créative puisqu'elle offre une approche innovante à l'étude de la langue. En effet, le traitement de données langagières réelles à travers l'utilisation des ordinateurs et des logiciels d'interrogation permet aux étudiants de focaliser leur attention sur des aspects spécifiques de la langue, de faire des hypothèses, et de devenir eux-mêmes des chercheurs aptes à faire des réflexions sur la langue et enfin de s'approprier la langue eux-mêmes (Aston). À ce propos, les laboratoires informatiques du Centre de Langues possèdent de nombreux outils d'interrogation de texte, mais pour cette expérimentation on a choisi SketchEngine. Ce logiciel performant permet l'extraction de plusieurs informations d'un corpus. ${ }^{15}$ Pour les objectifs de ce module trois outils ont été utilisés : la liste de mots, le concordancier, et le générateur de mots-clés. 
À l'aide de ces instruments, les étudiants ont généré deux listes de mots : l'une extraite de leurs corpus et l'autre extraite du corpus de référence. A ce moment-là, ils ont comparé les deux listes avec le générateur de mots-clés et obtenu une nouvelle liste, ou liste de mots-clés, qui montre, par ordre statistique, la saillance des mots contenus en premier lieu dans le corpus collecté et en même temps dans le corpus de référence. Sur la base des informations statistiques, mais également sur la base de leur propre curiosité, ils ont choisi dix mots ou termes à analyser.

Avec l'utilisation du concordancier, les étudiants ont tiré des exemples des dix items lexicaux choisis pour les observer et éventuellement identifier des éléments lexicaux complexes ou phraséologiques. Pour obtenir une vue d'ensemble sur l'usage en contexte des items, les étudiants les ont cherchés dans les corpus de référence où, encore une fois, ils ont collecté dix exemples. ${ }^{16}$

Avant de passer à l'utilisation de Trados, les étudiants ont traduit seuls les items choisis pour l'analyse ou, le cas échéant, en utilisant les dictionnaires bilingues en ligne (anglais, français et italiens) ou les moteurs de recherche. Les travaux ont été recueillis dans un dossier temporaire. A ce stade, on a fait une brève révision sur le logiciel Trados et ses outils, en particulier sur le MultiTerm et le Trados Studio, respectivement ayant la fonction de base de données terminologiques et d'environnement de traduction. Les étudiants ont d'abord ouvert le MultiTerm pour créer une base de données terminologique et insérer les dix items lexicaux choisis dans la langue source et la langue d'arrivée. ${ }^{17}$ Puis il leur a été demandé de choisir un texte parmi ceux précédemment collectés dans leurs corpus et puis de prendre en considération seulement un bref extrait. Le texte obtenu a été ouvert dans l'environnement de traduction et connecté avec la base des données terminologiques qu'ils avaient déjà créée.

\section{Bilan de l'expérimentation : le questionnaire}

Pour obtenir une vue d'ensemble et avoir des données pour comprendre la validité de l'expérience menée, plusieurs actions ont été entreprises. Tout d'abord, nous avons observé les étudiants pendant leurs activités tout en nous déplaçant entre les postes de travail PC. Pendant le déplacement, nous avons répondu aux questions et, en même temps, nous leur en avons posé pour mieux comprendre le déroulement des activités menées. En outre, pour obtenir plus de données, nous avons interviewé les étudiants pendant l'examen oral pour demander des précisions, pour mieux expliciter les réponses données dans le questionnaire soumis et pour ajouter de nouvelles informations. 
Le questionnaire proposé (cf. Annexe 1 et 2) était composé de deux parties : l'une contenant des questions sur les compétences acquises et l'autre sur les activités de l'analyse linguistique menée pendant le module.

\section{L’analyse des réponses : première partie du questionnaire}

L'analyse des réponses dans la première partie du questionnaire met en évidence quelques données intéressantes sur les compétences digitales initiales des étudiants. En particulier, les questions sur l'utilisation d'Internet ont signalé que certains étudiants ne peuvent pas encore effectuer des recherches avancées par l'intermédiaire des moteurs de recherche. Parmi les moteurs de recherche, la plupart des étudiants utilisent Google. En général, les étudiants sont aussi capables de télécharger des textes et des images et connaissent les outils pour les échanger, partager ou momentanément sauvegarder dans des espaces de stockage en ligne tels que DropBox, GoogleDrive. Les réponses aux questions ont aussi montré que les étudiants utilisent aussi des réseaux sociaux comme Facebook, Twitter, et Google +, Linkedin et en même temps ils peuvent utiliser les blogs, notamment Blogger.

Les questions sur la gestion du bureau et l'utilisation de logiciels ont révélé que les étudiants peuvent effectuer des opérations fondamentales, par exemple créer un dossier et un fichier, les renommer et les déplacer. La plupart des étudiants peuvent utiliser les logiciels du package Microsoft Office d'une façon presque avancée et seulement quelques-uns ne savent tout simplement pas comment créer des tables en Word et des calculs en Excel. Ils savent comment utiliser le PowerPoint de manière avancée par importation d'images, de musiques et de vidéos qui leur servira à exprimer leur créativité pour les tâches qui leur sont confiées. Au contraire, aucun étudiant ne sait gérer le logiciel Publisher, alors que la plupart connaît d'autres logiciels plus complexes utilisés pour éditer et monter des images et des vidéos (Windows Movie Maker et Photoshop).

\section{L'analyse des réponses : deuxième partie du questionnaire}

La deuxième partie du questionnaire est liée aux activités conduites par les étudiants pendant le module. Étant donné que la première question était sur le choix du site à partir duquel télécharger les textes nécessaires pour la construction d'un corpus de contenus institutionnels, les autres questions ont révélé des données intéressantes. En premier lieu, seuls trois étudiants ont employé beaucoup de temps pour trouver le sujet d'intérêt et ceci pour deux raisons, à savoir qu'ils n'étaient pas familiarisés avec ces sites, ou parce qu'ils ont mal choisi le sujet.

Une majorité d'étudiants a trouvé un nombre suffisant de textes pour la construction du corpus. Dans certains cas, où le sujet n'était pas suffisamment étendu, les étudiants ont 
rencontré des difficultés au point de changer de sujet ou d'élargir à d'autres sujets afférents et d'arriver à un nombre suffisant de textes.

En ce qui concerne l'analyse du lexique, les questionnaires ont montré que les étudiants ont préféré limiter l'analyse à des éléments lexicaux simples, par exemple, pauvreté, chômage, gouvernance, et reato, informazioni, confisca, pour les étudiants français. Un seul étudiant a opté pour le choix d'un groupe de mots ou de collocations, notamment économie verte, développement durable. Par ailleurs, tous ont fourni les occurrences les plus fréquentes trouvées dans leurs corpus et dans le corpus de référence. Ils ont aussi précisé s'ils ont trouvé des différences d'usage des éléments choisis pour l'analyse entre les deux corpus.

De la question 7 à la question 13, la focalisation se situe sur la traduction. En particulier, par la question numéro 7, les étudiants ont réfléchi sur les éléments linguistiques choisis pour fournir les solutions possibles et utiles à la médiation des sens. Après cette activité, il leur a été demandé de contrôler leurs traductions sur Internet et certains ont relevé l'impossibilité de trouver une correspondance, par exemple pour le mot gouvernance. Les étudiants ont dû aussi fournir des exemples d'usage, des éléments linguistiques choisis, sur Internet.

Pour mieux sensibiliser sur la complexité du processus de la traduction et sur les outils du traducteur, des activités ont été comprises dans les questions 10, 11, et 12. D'abord, tous les étudiants ont transcrit l'extrait du texte qu'ils avaient choisi pour la traduction en utilisant Trados (10). Puis, on leur a demandé de traduire le même texte par le logiciel Google Translator et de transcrire le résultat obtenu (11). Enfin, on leur a demandé de commenter les résultats obtenus. Dans tous les cas, les étudiants ont signalé les limitations de la traduction automatique donnant des explications basées sur le texte considéré. La question 13 a permis aux étudiants de s'exprimer sur l'expérience conduite et de réfléchir sur l'utilisation d'un logiciel de traduction assistée par ordinateur. Tous les étudiants ont mis en évidence les avantages d'un système constitué par plusieurs modules qui interagissent et fournissent aux traducteurs les outils indispensables pour se concentrer sur la traduction et non sur le repérage des informations à travers la lecture du texte papier.

Les deux dernières questions du questionnaire (14 et 15) se sont focalisées sur l'efficacité de cette expérimentation selon les étudiants. Dans tous les cas, ils ont exprimé un jugement positif aussi bien sur l'utilisation des outils présentés et utilisés pendant le module que sur les résultats obtenus au terme de la formation. L'expérience proposée leur a permis, comme ils l'indiquent dans les réponses, de connaître de nouveaux outils informatiques finalisés au traitement de la L2, en mesure de stimuler leur curiosité et développer de nouveaux savoirs ; en particulier les étudiants ont été sensibles à la découverte de la traduction dite 
automatique, mais qui de fait est assistée, permettant ainsi une participation authentique et créative du traducteur dans des contextes professionnels (entreprises, organismes institutionnels, etc.). Certains d'entre eux auraient souhaité prolonger le module en vue d'obtenir une pleine maitrise des usages numériques pour la traduction (comme la certification Trados fréquemment demandée par les entreprises).

\section{Conclusion}

L'expérimentation menée pendant le cours de FLE a montré que la créativité s'est manifestée à plusieurs niveaux et sous différentes formes. D’abord, elle est représentée par la capacité du professeur de réélaborer les ressources disponibles d'une manière innovante qui sache susciter chez l'apprenant une «émotion de la découverte». L'intégration de la linguistique de corpus avec la traduction assistée par ordinateur offre aux apprenants la possibilité de percevoir la L2 différemment, de manière active à travers le numérique.

Ensuite, la créativité s'est manifestée chez les apprenants lorsqu'ils ont trouvé une ou plusieurs solutions aux problèmes posés. Malgré les difficultés rencontrées dans l'utilisation des outils informatiques, le soutien et la présence d'un guide sont absolument nécessaires pour surmonter une attitude parfois réticente et promouvoir l'acquisition de compétences manquantes. L'ordinateur devient l'instrument qu'on doit connaitre pour travailler, pour trouver des réponses et obtenir des informations. L'attention portée nécessairement sur les difficultés issues d'un projet à la fois linguistique et informatique constitue une ressource qui a aidé les apprenants à mieux développer leurs compétences informatiques indispensables pour l'acquisition de la L2 au niveau avancé, en contexte professionnel tel qu'il est exigé aujourd'hui.

Enfin, Les activités conduites en classe ont montré que grâce à l'utilisation d'un logiciel spécifique, les étudiants peuvent aborder l'exécution d'un travail d'une façon organisée, par étapes liées à la logique spécifique des ordinateurs. Les compétences acquises leur ont permis de récupérer des textes, de développer leurs sujets de recherche, ou de choisir, d'une façon raisonnée, les mots à analyser en consultant une liste de mots présentée par ordre statistique. Ils ont aussi réussi à choisir des exemples d'usage pour les éléments lexicaux sélectionnés à partir des corpus qu'ils avaient constitués.

Pour mettre un bémol à l'ensemble de ce «montage » didactique-informatique, il est nécessaire de faire quelques considérations d'ordre technique : la réalisation des activités pour ce module a demandé beaucoup d'efforts pour la préparation des matériaux. La création d'un corpus pour la langue française et italienne a été complexe en ce qui concerne le choix des 
textes. ${ }^{18}$ À ce propos, la création d'un corpus de référence équilibré pour la langue française, où ne figureraient pas uniquement des textes de presse, doit être prise en considération, même si les objectifs initiaux de ce module étaient d'ordre méthodologique et non pas purement linguistique.

Toutefois, les commentaires positifs des étudiants permettent d'envisager le renouvellement de l'expérience de ce module en y apportant des modifications de rythme. Il serait approprié de revoir les unités didactiques, en particulier, celles consacrées à la terminologie et à sa réalisation.

En conclusion, on peut se demander si la «manipulation» de la langue dans un environnement numérique développe une capacité d'adaptation dans un monde en évolution et si l'intégration de deux approches développe une créativité indispensable pour travailler à l'international (communication et médiation linguistique et culturelle). «Etre créatif, [...] c'est imaginer des liens nouveaux entre des éléments connus » (Aden 5).

\section{Bibliographie}

Aden, Joëlle. «La créativité et l'apprentissage des langues ». Educateur 2 (2014) : 5.

Aston, Guy. «Applied Corpus Linguistics and the Learning Experience». Perspectives on Corpus Linguistics. Dir. Vander Viana, Sonia Zuengler et Geoff Barnbrook, Amsterdam : Benjamins, 2011. 1-16.

Bonnardel, Nathalie. «Entrée : Créativité ». Dictionnaire des Sciences Cognitives. Dir. G. Tiberghien. Paris : Colin/VUEF Armand, 2002. 95-97.

Cachia, Romina et al. «Creative Learning and Innovative Teaching Final Report on the Study on Creativity and Innovation in Education in the EU Member States ». JRC Technical Notes (2010) : 15. http://ftp.jrc.es/EURdoc/JRC62370.pdf 2010

Csikszentmihalyi, Mihaly. Creativity, the Psychology of Discovery and Invention. New York : Harper Collins, 1996.

Department for Education and Skills. The National Curriculum Handbook. Londres : Crown, 2004.

Edwards, Anthony. New Technology and Education. Londres : Continuum, 2012.

Ferrari, Anusca, Romina Cachia et Yves Punie. «Innovation and Creativity in Education and Training in the EU Member States : Fostering Creative Learning and Supporting Innovative Teaching ». JCR Technical Notes, 2009.

Hirschsprung, Nathalie. Apprendre et enseigner avec le multimédia. Paris : Hachette français langue étrangère, 2005. 
Hunston, Susanne. Corpora in Applied Linguistics. Cambridge : CUP, 2002.

Kozbelt, Aaron, Ronald A. Beghetto et Mark A. Runco. «Theories of Creativity ». Dir. James C. Kaufman et Robert J. Sternberg. The Cambridge Handbook of Creativity. Cambridge UP, 2010. 20-47.

Lankshear, Colin et Michele Knobel. New Literacies. Everyday Practices and Social Learning. New York : Open UP, 2011.

Parlement Européen et Conseil. Le Cadre européen des compétences Clés. En ligne http://eurlex.europa.eu/legal-ontent/FR/TXT/PDF/?uri=CELEX:32006H0962\&from=FR, 2006 Pugliese, Chaz. «Let Creativity into the Language Class». The Guardian en ligne http://www.theguardian.com/education/2012/mar/13/creativity-in-language-classroom Silverstone, Roger. Media and Morality : on the Rise of the Mediapolis. Cambridge : Cambridge UP, 2013.

Taddéi, François. «Inventer une nouvelle maïeutique pour apprendre à apprendre ». Cahiers pédagogiques 478 (2010) :17-44.

\footnotetext{
NOTES

${ }^{1}$ Adriana T. Damascelli est l'auteure de « Méthodologie expérimentale », «Bilan de l'expérimentation : le questionnaire », «Bibliographie », et « Annexes 1et $2 »$.

${ }^{2}$ Marie-Berthe Vittoz est l'auteure d' «Introduction », « Cadre théorique » et «Conclusions ».

${ }^{3}$ France. Ministère de l'éducation nationale e Ministère de la recherche (2000-2002), TICE: les technologies de l'information et de la communication pour l'enseignement dans les nouveaux programmes du lycée : mathématiques 1[ère] L, Reims : CRDP de Champagne-Ardenne, 2000

${ }^{4}$ Le processus est l'ensemble des mécanismes cognitifs qui sont à l'arrière de la pensée créative. Le produit se réfère au résultat du processus de la créativité, l'individu est considéré par ses habitudes intellectuelles, par exemple ouverture d'esprit ou compétence. Enfin, le lieu représente les situations dans lesquelles la créativité se développe.

${ }^{5}$ « ...l'école doit fournir aux élèves une éducation qui leur permettra de s'adapter à un environnement de plus en plus mondialisé, compétitif, diversifié et complexe, où la créativité, la capacité d'innover, le sens d'initiative, l'esprit d'entreprise et un engagement à poursuivre l'apprentissage sont tout aussi importants que la connaissance spécifique d'un sujet donné. » ${ }^{6}$ Sur le site de l'Union Européenne on lit: «Par la présente, la Commission proclame 2009 l'«Année européenne de la créativité et de l'innovation». Cette initiative vise à promouvoir les capacités de créativité et d'innovation en tant que compétences clés pour tous grâce à l'éducation et à la formation tout au long de la vie. Le défi consiste à instaurer un environnement propice à toutes les formes de créativité et d'innovation, qu'elles soient artistiques, culturelles, sociales ou technologiques, et à favoriser l'utilisation pratique des connaissances et des idées.

${ }^{7}$ Décision no 1350/2008/CE, en ligne http://eur-lex.europa.eu/LexUriServ/LexUriServ.do?uri=OJ:L:2008:348:0115:0117:FR:PDF

${ }^{8}$ Les descripteurs de Dublin sont: 1. Connaissances et compréhension, 2. Application des connaissances, 3. Analyse critique, 4. Communication, 5. Autodidaxie.

${ }^{9}$ Un corpus peut être exploré à l'aide d'outils d'analyse spécifiques dont le plus connu est le concordancier. Cet outil permet de rechercher dans un corpus toutes les occurrences d'un mot donné. Les résultats obtenus sont affiché sous forme de lignes contenant le mot-clé, qui, est mis en évidence.

${ }^{10} \mathrm{La}$ localisation linguistique est un processus de transfert et d'adaptation culturelle d'un produit (à des pays, régions ou groupes spécifiques) afin de prendre en compte les spécificités de deux marchés distincts. Elle implique l'étude exhaustive de la culture cible afin d'adapter de la manière la plus appropriée le produit aux besoins locaux.

${ }^{11}$ D'autres outils sont aussi disponibles et permettent, par l'exemple, de mettre les ressources telles que le mémoire de traductions et les bases de données terminologiques en commun et accessibles à la fois par plusieurs traducteurs engagés dans un projet de traduction.

${ }^{12}$ Ces étudiants ont le niveau $\mathrm{C} 1$ du CECR.

${ }^{13}$ Pour le français et l'italien on a collecté deux corpus de textes pris de différents journaux et concernant plusieurs sujets pour un total d'environ 100000 mots. Pour l'anglais, on a utilisé un corpus de langue anglaise britannique déjà existant le FLOB (Freiburg-London Bergen) d'environ un million de mots et caractérisé par des textes de langue anglaise britannique datant du début des années 1990.
} 
${ }^{14}$ Pour les étudiants avec des difficultés technologiques, des lignes directrices ont été données. En général ces informations concernaient l'enregistrement des documents collectés, la transformation en texte simple sans trace de mise en forme, et l'insertion, dans le corps du texte, des informations relatives à l'adresse Internet source du document.

${ }^{15}$ Ces logiciels permettent aussi de mener des recherches statistiques surtout exploitées par les lexicographes qui observent la distribution et l'utilisation d'un mot ou de certains phénomènes phraséologiques dans la langue. On en trouve de gratuits sur Internet.

${ }^{16}$ Etant donné que les textes des corpus collectés par les étudiants sont des sites institutionnels, la recherche dans un corpus de référence devrait donner comme résultat des occurrences disons « neutres » c'est-à-dire des occurrences différentes et liées à des utilisations générales, mais dans certains cas peut-être encore spécifiques, ou à d'autres sans rapport avec les occurrences déjà trouvées.

${ }^{17}$ En raison du temps relativement bref, les étudiants ont construit des glossaires contenant seulement la traduction des items choisis. On doit nécessairement préciser que MultiTerm, comme tous les outils professionnels pour la création de données terminologiques, permet d'insérer plusieurs informations afin que le traducteur puisse traduire sans difficulté.

${ }^{18}$ Dans le Centre de Langues de UniTO, les logiciels pour la langue française sont peu nombreux. De ce fait il a manqué aux étudiants un corpus de référence. 
Annexe 1. Questionnaire : première partie

UNIVERSITÀ

DEGLI STUDI

DI TORINO

DIPARTIMENTO DI LINGUE E LETTERATURE STRANIERE E CULTURE MODERNE

Laurea Magistrale in Lingue Straniere per la Comunicazione Internazionale LM-38

Lingua Francese - Prof.ssa Marie-Berthe Vittoz

a.a. 2013-2014

MODULE 3

LES TECHNOLOGIES POUR L'ETUDE DE LA LANGUE DES INSTITUTIONS INTERNATIONALES

Dr. Adriana T. Damascelli et Prof. Marie-Berthe Vittoz - Centro Linguistico di Ateneo (CLA-UNITO)

QUESTIONNAIRE

Merci, de bien vouloir répondre aux questions suivantes

PREMIÈRE PARTIE - Vos compétences informatiques. Lesquelles ?

\section{INTERNET}

a. Je peux faire des recherches avancées sur l'Internet en utilisant les opérateurs Booléens (and/or/not), sur moteurs de recherche.

Oui $\square$; Non $\square$; Si non, comment faites-vous à limiter la recherche pour arriver à un résultat plus précis ? Expliquez-le brièvement :

b. Lesquels moteurs de recherche utilisez-vous ?

c. Je peux évaluer la validité des sources d'information et ses contenus
Oui $\square$;
Oui, ma avec quelques incertitudes $\square$
Non $\square$

d. Je peux télécharger des textes/ des images / d'autres documents que je trouve dans mes recherches Oui, tous $\square$;

Si non tous, spécifier des trois types lesquels:

Si d'autres documents spécifier quels types :

e. Je peux utiliser les Web 2.0 applications (blogs, social networks, etc.)

Oui $\square$, spécifier lequel/lesquels

Non $\square$

f. Je connais Dropbox ou GoogleDrive pour échanger, partager ou momentanément sauver des documents Oui $\square$; Non, j'en utilise d'autres

\section{GESTION DU DESKTOP ET UTILISATION DES LOGICIELS}

a. Je peux créer un dossier

Oui $\square$;

Non $\square$

b. Je peux créer un fichier

Oui $\square$; Non $\square$

c. Je peux renommer un dossier / un fichier

Oui $\square$;

Non $\square$

d. Je peux déplacer un dossier / un fichier
Oui $\square$;
Non $\square$

e. Quels sont les logiciels Microsoft Office que vous savez utiliser ?

\begin{tabular}{|c|c|c|c|}
\hline Word $\square$; & Je peux créer des tables & Oui $\square$; & Non $\square$ \\
\hline Excel $\square$; & Je peux faire des calculs & Oui $\square$; & Non $\square$ \\
\hline PowerPoint $\square$; & Je peux insérer des images, de la musique, ou des vidéos & Oui $\square$; & Non $\square$ \\
\hline Publisher $\square$; & Spécifier ce que vous savez faire & & \\
\hline
\end{tabular}

f. Connaissez-vous et savez-vous utilisez d'autres logiciels ? Spécifiez lequel/lesquels 
Annexe 2. Questionnaire : deuxième partie

UNIVERSITÀ

DEGLI STUD

DI TORINO

DIPARTIMENTO DI LINGUE E LETTERATURE STRANIERE E CULTURE MODERNE

Laurea Magistrale in Lingue Straniere per la Comunicazione Internazionale LM-38

Lingua Francese - Prof.ssa Marie-Berthe Vittoz

a.a. $2013-2014$

NOM

MODULE 3

LES TECHNOLOGIES POUR L'ETUDE DE LA LANGUE DES INSTITUTIONS INTERNATIONALES

Dr. Adriana T. Damascelli et Prof. Marie-Berthe Vittoz, Centro Linguistico di Ateneo (CLA-UniTO)

QUESTIONNAIRE

Merci, de bien vouloir répondre aux questions suivantes

DEUXIÈME PARTIE - Analyser la langue des sites institutionnels - résultats de votre recherche au CLA-UniTO

1. Quel site Internet avez-vous choisi pour votre recherche parmi ceux qui vous ont été signalés ? Pourquoi ? Indiquez l'adresse ci-dessous et justifiez votre choix

2. Pendant la consultation du site choisi, avez-vous trouvé immédiatement un sujet d'intérêt ? Lequel ? (Indiquez-le) Dans le cas contraire, quelle a été votre difficulté / quelles ont été vos difficultés?

3. Avez-vous trouvé un nombre suffisant de textes pour la construction du corpus ? Si non, quelle stratégie avez-vous adopté pour obtenir le matériel nécessaire?

4. Quels éléments linguistiques avez-vous choisi dans votre corpus pour votre analyse ? Eléments lexicaux simples ou complexes? Indiquez-les ci-dessous (au moins dix).

5. Quelles occurrences avez-vous choisi pour afficher les mots/les expressions en contexte ? Ecrivez-les et expliquez votre choix

6. Quelles sont les occurrences trouvées dans votre corpus de départ et celles que vous avez retrouvées dans le corpus de référence (site du CLA pour le français et l'italien et Lab A CLA pour l'anglais). Avez-vous trouvé des différences ? Pouvez-vous les copier ci-dessous puis essayez de donner une brève explication

7. Comment traduiriez-vous les mots/termes et/ou expressions trouvés ? Montrez les solutions proposées

8. Si vous avez traduit par vous-même, avez-vous contrôlé sur Internet s'il existe des correspondances entre vos solutions et celles qui sont données par les moteurs de recherche?

9. Ecrivez des exemples tirés d'Internet (au moins deux) pour chaque mot/terme ou expression choisi

10. Pour l'utilisation du logiciel TRADOS, vous avez choisi un morceau de texte parmi ceux que vous avez inclus dans votre corpus. Pouvez-vous transcrire le petit texte source et le texte traduit ?

11. Essayer de traduire le morceau de texte que vous avez traduit par TRADOS en utilisant « Google translator »

12. Quelles sont les différences que vous remarquez entre votre traduction et celle produite par \& Google translator *

13. Quelles sont les avantages d'utiliser un logiciel comme TRADOS ? (Pensez à l'existence ou création de mémoire de traduction et à la création ou consultation de banques terminologiques)

14. Pensez-vous que ce travail pourrait aider à réfléchir sur la langue et à son utilisation dans différents contextes ? De quelle façon?

15. En général, bien que le module ait été relativement bref, avez-vous trouvé ces activités intéressantes ? Justifiez brièvement votre réponse 